\title{
On the Micro-Hyperbolic Boundary Value Problem for Systems of Differential Equations
}

\author{
To the memory of Emmanuel Andronikof
}

By

Motoo UCHIDA*

\begin{abstract}
We formulate the boundary value problem for systems of linear differential equations which satisfy a certain condition of micro-hyperbolicity at the boundary in the same way as the Kashiwara-Kawai formulation for elliptic systems.
\end{abstract}

\section{Résumé}

Le problème au bord est formulé pour les systèmes des équations aux dérivées partiélles qui satisfont à une condition de micro-hyperbolicité, comme la formulation de Kashiwara-Kawai pour les systèmes elliptiques.

\section{$\S 0 . \quad$ Introduction}

In $[\mathrm{KK}]$, Kashiwara and Kawai formulated boundary value problems for elliptic systems of differential equations from a microlocal point of view. They described the cohomology groups of $\left.\mathrm{R} \Gamma_{Z_{+}} \mathrm{RHom} \mathcal{D}_{M}\left(\mathcal{M}, \mathcal{B}_{M}\right)\right|_{N}$, where $Z_{+}$is a closed domain in a manifold $M$ with boundary $N$ and $\mathcal{M}$ is an elliptic $\mathcal{D}_{M^{-}}$ module, in terms of a system of micro-differential equations induced on the boundary. In this paper we extend the Kashiwara-Kawai formula to systems of differential equations which satisfy a certain condition of micro-hyperbolicity

Communicated by T. Kawai, February 2, 2000. Revised October 30, 2000.

2000 Mathematics Subject Classification(s): 35G15.

Key words and phrases: Boundary value problem, D-module.

* Department of Mathematics, Graduate School of Science, Osaka University, Machikaneyama-Cho 1-16, Toyonaka, Osaka 560-0043, Japan.

e-mail: uchida@math.wani.osaka-u.ac.jp 
at the boundary. In this case, also, we are able to define a coherent $\mathcal{E}_{Y}$-module $\mathcal{N}^{+}$and prove

$$
\left.\mathrm{R} \Gamma_{Z_{+}} \mathrm{RHom} \mathcal{D}_{X}\left(\mathcal{M}, \mathcal{B}_{M}\right)\right|_{N}[1] \cong \mathrm{R} \dot{\pi}_{N *} \mathrm{RHom}_{\mathcal{E}_{Y}}\left(\mathcal{N}^{+}, \mathcal{C}_{N}\right)
$$

(see Theorem 1.2). It is also possible to microlocalize formula (0.1) by using the sheaf $\mathcal{C}_{Z_{+} \mid X}$ of Kataoka. By this, we can reformulate the main part of the works of Kaneko [Kn] and Oaku [O, Section 3] in the derived category (i.e., for all cohomology groups) of sheaves for systems of differential equations (see Section 3).

This paper is an enlarged version of [U].

Notation. In this paper, we freely use the notation of [KS1] for sheaves and functors. For a complex manifold $X, T^{*} X$ denotes the cotangent bundle of $X$, and $\dot{T}^{*} X$ that with the zero section removed. $\pi$ denotes the projection $T^{*} X \rightarrow X . \mathcal{O}_{X}$ denotes the sheaf of holomorphic functions on $X, \mathcal{D}_{X}$ the sheaf of rings of differential operators, and $\mathcal{E}_{X}$ the sheaf of rings of microdifferential operators (cf. [SKK]). If $M$ is a closed real submanifold of $X, T_{M}^{*} X$ denotes the conormal bundle of $M$. We often denote by $M$ the zero section of $T_{M}^{*} X$, and $\dot{T}_{M}^{*} X=T_{M}^{*} X \backslash M$. For a holomorphic function $f$ on $X$ and $p \in T^{*} X$, $d f(p)$ denotes $\pi^{*}(d f)$, with $\pi^{*}: T_{\pi(p)}^{*} X \hookrightarrow T_{p}^{*} T^{*} X$.

We denote by $H$ the Hamiltonian map $T^{*} T^{*} X \rightarrow T T^{*} X$. We identify $T T^{*} X \cong T\left(T^{*} X^{\mathbf{R}}\right), T^{*} X^{\mathbf{R}}$ being the underlying real manifold of $T^{*} X$. For subsets $V, W$ of $T^{*} X, C(V, W)$ denotes the Whitney normal cone, which is a closed conic subset of $T T^{*} X$ and considered also as a subset of $T^{*} T^{*} X$ by the isomorphism $-H: T^{*} T^{*} X \stackrel{\sim}{\rightarrow} T T^{*} X$.

\section{$\S 1 . \quad$ Main Result}

Let $M$ be a real analytic manifold of dimension $n \geq 1, N$ a submanifold of $M$ of codimension 1 defined by the equation $f=0$ for a real-valued analytic function $f$ with $\left.d f\right|_{N} \neq 0$. (We denote also by $f$ the holomorphic extension of $f$ to a complex neighborhood of $M$.) Let $X$ be a complex neighborhood of $M$, $Y$ a closed complex submanifold of $X$ of codimension 1 defined by $f=0$, and $\varphi$ the embedding $Y \hookrightarrow X$.

Let $Z_{+}$denote the closed subset $\{f \geq 0\}$ of $M$; then $Z_{+}$is a closed domain in $M$ with analytic boundary. We set $N^{+}=\{k d f(x) \mid x \in N, k>0\}$. We also set

$$
H=\left\{(x, \xi) \in T_{M}^{*} X \mid x \in N, \xi \neq 0\right\}\left(=N \times_{M} \dot{T}_{M}^{*} X\right) .
$$

Let $\mathcal{M}$ be a coherent $\mathcal{D}_{X}$-module. $\operatorname{Ch}(\mathcal{M})$ denotes the characteristic variety of $\mathcal{M}$. We assume the following two conditions: 
(a.1) $\varphi: Y \rightarrow X$ is non characteristic for $\mathcal{M}$;

(a.2) For all $p \in H$,

$$
d f(p) \notin C\left(\mathrm{Ch}(\mathcal{M}), Z_{+} \times_{M} T_{M}^{*} X\right) .
$$

Let $\left(T_{N}^{*} X\right)^{+}$be an open subset of $T_{N}^{*} X$ defined by $\left(T_{N}^{*} X\right)^{+}=q^{-1}\left(N^{+}\right)$, with $q$ being the canonical projection $T_{N}^{*} X \rightarrow T_{N}^{*} M$. Let ${ }^{t} \varphi^{\prime}: T^{*} X \times_{X} Y \rightarrow$ $T^{*} Y$ the induced map of $\varphi$, and $\rho: T_{N}^{*} X \rightarrow T_{N}^{*} Y$ the restriction of ${ }^{t} \varphi^{\prime}$ to $T_{N}^{*} X$ :

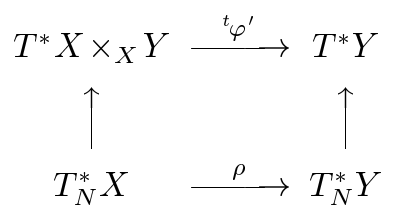

Let $\widetilde{\mathcal{M}}=\mathcal{E}_{X} \otimes_{\pi^{-1} \mathcal{D}_{X}} \pi^{-1} \mathcal{M}$, the $\mathcal{E}_{X}$-module associated to $\mathcal{M}$. Let $\varphi^{*} \widetilde{\mathcal{M}}$ denote the induced $\mathcal{E}_{Y}$-module of $\widetilde{\mathcal{M}}$ on $Y$. We define the $\mathcal{E}_{Y}$-module $\mathcal{N}^{+}$(on $\left.\dot{T}_{N}^{*} Y\right)$ by

$$
\mathcal{N}^{+}=\rho_{*}\left(\mathbf{C}_{\left(T_{N}^{*} X\right)^{+}} \otimes \mathcal{E}_{Y \rightarrow X} \otimes_{\mathcal{E}_{X}} \mathcal{M}\right)
$$

Then we have

Lemma 1.1. If we assume (a.1) and (a.2), $\mathcal{N}^{+}$is $\mathcal{E}_{Y}$-coherent on $\dot{T}_{N}^{*} Y$ and there is an $\mathcal{E}_{Y}$-linear homomorphism $\mathcal{N}^{+} \rightarrow \varphi^{*} \widetilde{\mathcal{M}}$ such that

$$
\mathcal{N}_{q}^{+} \cong \bigoplus_{p \in\left(T_{N}^{*} X\right)^{+} \cap \operatorname{Supp}(\widetilde{\mathcal{M}}) \cap \rho^{-1}(q)} \mathcal{E}_{Y \rightarrow X} \otimes_{\mathcal{E}_{X}} \widetilde{\mathcal{M}}_{p}
$$

for any $q \in T_{N}^{*} Y \backslash N$.

Remark. A coherent $\mathcal{E}_{Y^{-}}$-module $\mathcal{N}^{+}$with an $\mathcal{E}_{Y^{-}}$linear map $\alpha: \mathcal{N}^{+} \rightarrow$ $\varphi^{*} \widetilde{\mathcal{M}}$ satisfying (1.3) is unique up to isomorphisms. If $\left(\mathcal{N}_{1}^{+}, \alpha_{1}\right)$ and $\left(\mathcal{N}_{2}^{+}, \alpha_{2}\right)$ are such pairs, we have an isomorphism $\beta$ such that

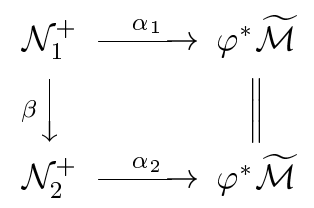

is commutative. 
Let $\mathcal{B}_{M}$ be the sheaf of hyperfunctions on $M, \mathcal{C}_{N}$ the sheaf of microfunctions on $N$ (cf. [SKK]). Let or $_{N \mid M}$ be the relative orientation sheaf of $N$ in $M$.

Theorem 1.2. Assume (a.1) and (a.2). There is an isomorphism

$$
\left.\mathrm{R} \Gamma_{Z_{+}} \mathrm{R} \mathcal{H} o m_{\mathcal{D}_{X}}\left(\mathcal{M}, \mathcal{B}_{M}\right)\right|_{N} \otimes \operatorname{or}_{N \mid M}[1] \cong \mathrm{R} \dot{\pi}_{N *} \mathrm{RHom}_{\mathcal{E}_{Y}}\left(\mathcal{N}^{+}, \mathcal{C}_{N}\right)
$$

where $\dot{\pi}_{N}: \dot{T}_{N}^{*} Y \rightarrow N$.

Corollary 1.3. Assume (a.1) and (a.2), and assume also $\mathcal{N}^{+}=0$. Letting $M_{+}=Z_{+} \backslash N$, we have the isomorphism

$$
\left.\mathrm{R}_{M_{+}} \mathrm{RH} \operatorname{mom}_{\mathcal{D}_{X}}\left(\mathcal{M}, \mathcal{B}_{M}\right)\right|_{N} \stackrel{\sim}{\longrightarrow} \operatorname{RHom}_{\mathcal{D}_{Y}}\left(\mathcal{M}_{Y}, \mathcal{B}_{N}\right)
$$

where $\mathcal{M}_{Y}$ is the induced $\mathcal{D}_{Y}$-module of $\mathcal{M}$.

(This follows from Theorem 1.2 and (3.5.8) of [SKK, Ch. II].)

Remark 1. Theorem 1.2 is first proved for elliptic $\mathcal{D}_{X}$-modules by Kashiwara and Kawai [KK]. Note that (a.1) and (a.2) are automatically satisfied if $\mathcal{M}$ is elliptic.

Remark 2. Condition (1.1) is an analogue of micro-hyperbolicity [KS2] and naturally appears in microlocal study of boundary value problems (cf. [S2, $\mathrm{SZ}]$ ). It is well known that, if we assume

$$
-d f(p) \notin C\left(\mathrm{Ch}(\mathcal{M}), Z_{+} \times_{M} T_{M}^{*} X\right)
$$

at $p \in T_{M}^{*} X \cap T_{N}^{*} X$, this entails propagation of regularity up to the boundary point $p$ from the positive side of $N$ (see [Kt2, S1, S2, SZ]).

Recall that $\varphi$ is micro-hyperbolic for $\mathcal{M}$ at $p \in H$ in the sense of [KS2, Definition 2.1.2] if

$$
\pm d f(p) \notin C\left(\mathrm{Ch}(\mathcal{M}), T_{M}^{*} X\right)
$$

for both \pm . Let $\mathcal{A}_{M}$ be the sheaf of real analytic functions on $M$.

Theorem 1.4. Assume that $\varphi: Y \rightarrow X$ is non characteristic for $\mathcal{M}$ and is micro-hyperbolic for $\mathcal{M}$ at all $p \in H$. Then there is an isomorphism

$$
\left.\mathrm{R} \Gamma_{Z_{+}} \mathrm{RHom}_{\mathcal{D}_{X}}\left(\mathcal{M}, \mathcal{A}_{M}\right)\right|_{N} \otimes \operatorname{or}_{N \mid M}[1] \cong \mathrm{R} \dot{\pi}_{N *} \operatorname{RHom}_{\mathcal{E}_{Y}}\left(\mathcal{N}^{+}, \mathcal{C}_{N}\right)
$$

as well as isomorphism (1.4), where $\mathcal{N}^{+}$is the coherent $\mathcal{E}_{Y}$-module defined by $(1.2)$. 
Example. Let $\left(x_{1}, \ldots, x_{n}\right)$ be a system of local coordinates of $M, Z_{+}=$ $\left\{x_{1} \geq 0\right\}$. Let $D_{i}=\partial / \partial x_{i}, i=1, \ldots, n, D^{\prime}=\left(D_{2}, \ldots, D_{n}\right)$, and let

$$
P(x, D)=D_{1}^{2} I_{r}-\left\{A\left(x, D^{\prime}\right)+x_{1}^{3} B\left(x, D^{\prime}\right)\right\},
$$

where $I_{r}$ denotes the $r \times r$ identity matrix, $A\left(x, D^{\prime}\right)$ is an $r \times r$ matrix of differential operators of order 2 of which the principal symbol $\sigma_{2}(A)\left(x, i \eta^{\prime}\right)$ is a negative semi-definite hermitian matrix on $T_{M}^{*} X$ and so is $B\left(x, D^{\prime}\right)$. Let $\mathcal{M}=\mathcal{D}_{X}^{r} / \mathcal{D}_{X}^{r} P$; then $\mathcal{M}$ satisfies (a.1) and (a.2), and $\mathcal{N}^{+}=0$.

\section{$\S 2 . \quad$ Proof of Theorems 1.2 and 1.4}

As in [KK], the proof of Theorem 1.2 is divided into two steps. In the first step, we relate the left-hand side of (1.4) to a differential complex with coefficients in $\mathcal{C}_{N \mid X}$ induced from $\mathcal{M}$. In the second step, proving Lemma 1.1, we complete the proof of Theorem 1.2.

Let us recall the notion of the $\mathcal{E}_{X^{-} \text {-module }} \mathcal{C}_{Z_{+} \mid X}$ due to Kataoka [Kt1] and Schapira [S2]. Following [S2], let

$$
\mathcal{C}_{Z_{+} \mid X}=\mu \operatorname{hom}\left(\mathbf{C}_{Z_{+}}, \mathcal{O}_{X}\right) \otimes \operatorname{or}_{M \mid X}[n] .
$$

Then all the cohomology groups $H^{k}\left(\mathcal{C}_{Z_{+} \mid X}\right), k \neq 0$, are zero and $H^{0}\left(\mathcal{C}_{Z_{+} \mid X}\right)$ is an $\mathcal{E}_{X^{-}}$module. We identify $\mathcal{C}_{Z_{+} \mid X}$ with its zero-th cohomology $H^{0}\left(\mathcal{C}_{Z_{+} \mid X}\right)$. For the $\mathcal{E}_{X}$-module $\mathcal{C}_{N \mid X}$, refer to [KK], [KS2] and also [S1, S2]. (In this paper, we follow the definition of $[\mathrm{KK}, \mathrm{KS} 2]: \mathcal{C}_{N \mid X}=H^{n} \mu_{N}\left(\mathcal{O}_{X}\right) \otimes$ or $\left._{N \mid X}.\right)$ We prepare two lemmas.

\section{Lemma 2.1.}

(1) $\left.\mathrm{R} \pi_{*} \mathcal{C}_{Z_{+} \mid X}\right|_{M} \cong \mathrm{R} \Gamma_{Z_{+}} \mathcal{B}_{M}$.

(2) $\operatorname{supp}\left(\mathcal{C}_{Z_{+} \mid X}\right) \cap T_{N}^{*} X \subset \overline{\left(T_{N}^{*} X\right)^{+}}$.

(3) There is an $\mathcal{E}_{X}$-linear homomorphism $\mathcal{C}_{N \mid X} \otimes$ or $_{N \mid M} \rightarrow \mathcal{C}_{Z_{+} \mid X}$, and this is an isomorphism on $\left(T_{N}^{*} X\right)^{+}$.

For the proof, see [Kt3, Section 4] and [S2, S3].

Lemma 2.2. If we assume (1.1) at a point $p$ of $T_{M}^{*} X \cap T_{N}^{*} X$, we have

$$
\left.\operatorname{RHom}_{\mathcal{E}_{X}}\left(\widetilde{\mathcal{M}}, \mathcal{C}_{Z_{+} \mid X}\right)\right|_{T_{N}^{*} X}=0
$$

in a neighborhood of $p$. 
Proof. (Cf. the proof of Corollary 3.3 of [SZ].) Let $g$ be a real-valued smooth function defined on $X$ such that $\left.g\right|_{M}=f$. We set $h=g \circ \pi$, with $\pi: T^{*} X \rightarrow X$. From (1.1), we have

$$
d h \notin C_{p}\left(\mathrm{Ch}(\mathcal{M}), Z_{+} \times_{M} T_{M}^{*} X\right) .
$$

Hence we can find an open subset $U$ of $T^{*} X$ so that $U \cap \operatorname{Ch}(\mathcal{M})=\varnothing$,

$$
d h \notin C_{p}\left(T^{*} X \backslash U, Z_{+} \times_{M} T_{M}^{*} X\right),
$$

and $d h \notin C_{p}\left(T^{*} X \backslash U, U\right)$. Let $\operatorname{SS}\left(Z_{+}\right)$denote, for simplicity, the micro-support $\operatorname{SS}\left(\mathbf{C}_{Z_{+}}\right)$of the sheaf $\mathbf{C}_{Z_{+}}$on $X$ (cf. [KS1, Section 5.1]). Since

$$
\operatorname{SS}\left(Z_{+}\right) \subset\left(Z_{+} \times_{M} T_{M}^{*} X\right) \cup U
$$

in a neighborhood of $p$, we have $d h \notin C_{p}\left(T^{*} X \backslash U, \operatorname{SS}\left(Z_{+}\right)\right)$. This yields

$$
d h \notin C_{p}\left(\mathrm{Ch}(\mathcal{M}), \operatorname{SS}\left(Z_{+}\right)\right) .
$$

Since

$$
\operatorname{SS}\left(\operatorname{RHom} \mathcal{D}_{X}\left(\mathcal{M}, \mathcal{C}_{Z_{+} \mid X}\right)\right) \subset C\left(\mathrm{Ch}(\mathcal{M}), \operatorname{SS}\left(Z_{+}\right)\right),
$$

it follows from the definition of micro-supports that

$$
\left.\mathrm{R} \Gamma_{\{h \geq 0\}} \mathrm{RHom}_{\mathcal{D}_{X}}\left(\mathcal{M}, \mathcal{C}_{Z_{+} \mid X}\right)\right|_{\{h=0\}}=0
$$

in a neighborhood of $p$. Since $\mathcal{C}_{Z_{+} \mid X}$ is supported on $\operatorname{SS}\left(Z_{+}\right)$and $\operatorname{SS}\left(Z_{+}\right) \subset$ $\{h \geq 0\}$, we have

$$
\left.\mathrm{RHom}_{\mathcal{D}_{X}}\left(\mathcal{M}, \mathcal{C}_{Z_{+} \mid X}\right)\right|_{\{h=0\}}=0 .
$$

Since $\mathbf{C}_{Z_{+}}$is cohomologically constructible, if we set

$$
F=\operatorname{RHom}_{\mathcal{D}_{X}}\left(\mathcal{M}, \mathcal{O}_{X}\right),
$$

it follows from [KS1, Proposition 4.4.2] that

$$
\begin{aligned}
\left.\mathrm{R} \pi_{*} \mathrm{R}_{T_{X}^{*} X} \mathrm{RH} \operatorname{lom}_{\mathcal{D}_{X}}\left(\mathcal{M}, \mathcal{C}_{Z_{+} \mid X}\right)\right|_{N} & \left.\cong \mathrm{R} \pi_{*} \mathrm{R}_{T_{X}^{*} X} \mu \operatorname{hom}\left(\mathbf{C}_{Z_{+}}, F\right)\right|_{N}[n] \\
& \left.\cong F \otimes \operatorname{RHom}_{\mathbf{C}}\left(\mathbf{C}_{Z_{+}}, \mathbf{C}_{X}\right)\right|_{N}[n] \\
& \left.\cong F \otimes \mathbf{C}_{M_{+}}\right|_{N} \\
& =0
\end{aligned}
$$

where we set $M_{+}=Z_{+} \backslash N$. Hence, from Lemma 2.1, we have

$$
\begin{aligned}
\left.\mathrm{R}_{Z_{+}} \mathrm{RHom}_{\mathcal{D}_{X}}\left(\mathcal{M}, \mathcal{B}_{M}\right)\right|_{N} & \left.\cong \mathrm{R} \pi_{*} \mathrm{RHom}_{\mathcal{D}_{X}}\left(\mathcal{M}, \mathcal{C}_{Z_{+} \mid X}\right)\right|_{N} \\
& \left.\cong \mathrm{R} \pi_{*} \mathrm{R} \Gamma_{T^{*} X \backslash X} \mathrm{RHom} \mathcal{D}_{\mathcal{D}_{X}}\left(\mathcal{M}, \mathcal{C}_{Z_{+} \mid X}\right)\right|_{N} \\
& \cong \mathrm{R} \pi_{*}^{\prime}\left(\left.\mathrm{RHom}_{\mathcal{E}_{X}}\left(\widetilde{\mathcal{M}}, \mathcal{C}_{Z_{+} \mid X}\right)\right|_{T_{N}^{*} \backslash \backslash N}\right),
\end{aligned}
$$


where $\pi^{\prime}: \dot{T}_{N}^{*} X \rightarrow N$. It then follows from Lemmas $2.1(2),(3)$ and 2.2 that

$$
\begin{aligned}
\left.\operatorname{RHom}_{\mathcal{E}_{X}}\left(\widetilde{\mathcal{M}}, \mathcal{C}_{Z_{+} \mid X}\right)\right|_{T_{N}^{*} X \backslash N} & \cong \mathrm{R} \Gamma_{\left(T_{N}^{*} X\right)+}\left(\left.\operatorname{RHom}_{\mathcal{E}_{X}}\left(\widetilde{\mathcal{M}}, \mathcal{C}_{Z_{+} \mid X}\right)\right|_{T_{N}^{*} X \backslash N}\right) \\
& \cong \mathrm{R} \Gamma_{\left(T_{N}^{*} X\right)}+\operatorname{RHom}_{\mathcal{E}_{X}}\left(\widetilde{\mathcal{M}}, \mathcal{C}_{N \mid X}\right) \otimes \operatorname{or}_{N \mid M}
\end{aligned}
$$

Thus we have

$$
\begin{aligned}
\left.\mathrm{R} \Gamma_{Z_{+}} \mathrm{RHom}_{\mathcal{D}_{X}}\left(\mathcal{M}, \mathcal{B}_{M}\right)\right|_{N} & \otimes \operatorname{or}_{N \mid M} \\
& \cong \mathrm{R} \pi_{*}^{\prime} \mathrm{R} \Gamma_{\left(T_{N}^{*} X\right)^{+}} \mathrm{RHom}_{\mathcal{E}_{X}}\left(\widetilde{\mathcal{M}}, \mathcal{C}_{N \mid X}\right)
\end{aligned}
$$

Since $T_{Y}^{*} X \cap \operatorname{Supp}(\widetilde{\mathcal{M}}) \subset T_{X}^{*} X$, we have

$$
\begin{aligned}
\mathrm{RHS} \text { of }(2.1) & \cong \mathrm{R} \dot{\pi}_{N *} \mathrm{R} \rho_{*} \mathrm{R} \Gamma_{\left(T_{N}^{*} X\right)^{+}} \mathrm{RHom} \mathcal{E}_{X}\left(\widetilde{\mathcal{M}}, \mathcal{C}_{N \mid X}\right) \\
& =\mathrm{R} \dot{\pi}_{N *} \mathrm{R} \rho_{*}^{+}\left(\left.\mathrm{RHom}_{\mathcal{E}_{X}}\left(\widetilde{\mathcal{M}}, \mathcal{C}_{N \mid X}\right)\right|_{\left(T_{N}^{*} X\right)^{+}}\right),
\end{aligned}
$$

where we denote by $\rho^{+}:\left(T_{N}^{*} X\right)^{+} \rightarrow T_{N}^{*} Y$ the restriction of $\rho$. Hence, in summary, we have

$$
\begin{aligned}
\left.\mathrm{R}_{Z_{+}} \operatorname{RHom}_{\mathcal{D}_{X}}\left(\mathcal{M}, \mathcal{B}_{M}\right)\right|_{N} & \otimes \operatorname{or}_{N \mid M} \\
& \cong \mathrm{R} \dot{\pi}_{N *} \operatorname{R} \rho_{*}^{+}\left(\left.\operatorname{RHom}_{\mathcal{E}_{X}}\left(\widetilde{\mathcal{M}}, \mathcal{C}_{N \mid X}\right)\right|_{\left(T_{N}^{*} X\right)^{+}}\right)
\end{aligned}
$$

In the rest of this section, we prove

$$
\mathrm{R} \rho_{*}^{+}\left(\left.\operatorname{RHom}_{\mathcal{E}_{X}}\left(\widetilde{\mathcal{M}}, \mathcal{C}_{N \mid X}\right)\right|_{\left(T_{N}^{*} X\right)^{+}}\right)[1] \cong \operatorname{RHom}_{\mathcal{E}_{Y}}\left(\mathcal{N}^{+}, \mathcal{C}_{N}\right)
$$

on $T_{N}^{*} Y \backslash N$. Combining (2.2) and (2.3), we get isomorphism (1.4).

We prepare two lemmas for the second part of the proof. Lemma 1.1 follows from the following Lemma 2.3 with $\Omega=T_{N}^{*} Y \backslash N$.

Lemma 2.3. Let $\Omega$ be a conic open subset of $T_{N}^{*} Y \backslash N$. Let $\rho: T_{N}^{*} X \rightarrow$ $T_{N}^{*} Y$, and let $\mathcal{M}$ be a coherent $\mathcal{E}_{X}$-module on a conic neighborhood of $\rho^{-1}(\Omega)$. Assume

(1) $\varphi: Y \rightarrow X$ is non characteristic for $\mathcal{M}$ on a neighborhood of $\Omega$ in the sense of [SKK, Ch. II, Def. 3.5.4].

(2) For a conic neighborhood $U$ of $\rho^{-1}(\Omega) \cap T_{M}^{*} X, U \cap\left(T_{N}^{*} X\right)^{+} \cap \operatorname{Supp}(\mathcal{M})=\varnothing$. Then $\rho$ is finite on $\rho^{-1}(\Omega) \cap\left(T_{N}^{*} X\right)^{+} \cap \operatorname{Supp}(\mathcal{M})$, and, if we set

$$
\mathcal{N}^{+}=\rho_{*}\left(\mathbf{C}_{\left(T_{N}^{*} X\right)^{+}} \otimes \mathcal{E}_{Y \rightarrow X} \otimes_{\mathcal{E}_{X}} \mathcal{M}\right)
$$

$\mathcal{N}^{+}$is a coherent $\mathcal{E}_{Y}$-module over $\Omega$. 
Proof. By hypothesis (1), we may assume that $\mathcal{M}$ is a coherent $\mathcal{E}_{X^{-}}$ module defined on ${ }^{t} \varphi^{\prime-1}(V)$ for an open conic neighborhood $V$ of $\Omega$ in $\dot{T}^{*} Y$. In order to prove the coherency of $\mathcal{N}^{+}$locally on $\Omega$, by taking a local coordinate system $\left(z_{1}, z^{\prime}\right)$ of $X$ which is real on $M$ and such that $Z_{+}=M \cap\left\{z_{1} \geq 0\right\}$, we identify $T^{*} X \times_{X} Y=T^{*} Y \times \mathbf{C}_{\tau}$ and ${ }^{t} \varphi^{\prime}$ with the projection to $T^{*} Y$. Then

$$
\begin{gathered}
{ }^{t} \varphi^{\prime-1}(V)=V \times \mathbf{C}_{\tau}, \\
{ }^{t} \varphi^{\prime-1}(V) \cap\left(T_{N}^{*} X\right)^{+}=\Omega \times\{\operatorname{Re} \tau>0\} .
\end{gathered}
$$

It then follows from the assumption (1) and (2) that, for any $q \in \Omega$, there exist a neighborhood $V_{q}$ of $q$ in $V$ and $\delta>0$ such that $\mathcal{M}=0$ on $\left(V_{q} \cap \Omega\right) \times\{\tau \mid 0<$ $\operatorname{Re} \tau<\delta$. Again by (1), by making $V_{q}$ smaller enough if necessary, we may assume also that $\mathcal{M}=0$ on $V_{q} \times\{\tau \mid \operatorname{Re} \tau=\delta / 2\}$. Let us set

$$
W=V_{q} \times \mathbf{C}_{\tau} \quad \text { and } \quad W^{\prime}=V_{q} \times\{\tau \mid \operatorname{Re} \tau>\delta / 2\} ;
$$

they are open subsets of $T^{*} X \times{ }_{X} Y$. Then ${ }^{t} \varphi^{\prime}$ is proper on $W^{\prime} \cap \operatorname{Supp}(\mathcal{M})$, and

$$
\operatorname{Supp}(\mathcal{M}) \cap W \cap\left(T_{N}^{*} X\right)^{+}=\operatorname{Supp}(\mathcal{M}) \cap W^{\prime} \cap \rho^{-1}(\Omega) .
$$

Hence $\mathcal{N}^{+}={ }^{t} \varphi_{*}^{\prime}\left(\left.\mathcal{E}_{Y \rightarrow X} \otimes_{\mathcal{E}_{X}} \mathcal{M}\right|_{W^{\prime}}\right)$ on $V_{q} \cap \Omega$, and it follows from Theorem 3.5.3 of [SKK, Ch. II] that the right-hand side is a coherent $\mathcal{E}_{Y}$-module.

Q.E.D.

Lemma 2.4. Let $\mathcal{M}$ be as in Lemma 2.3. Then there exists a commutative diagram on $\Omega$

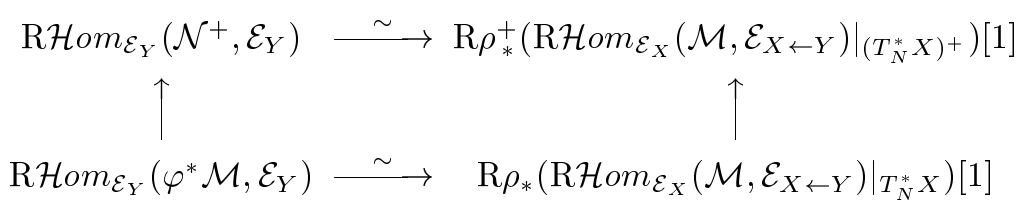

and every horizontal arrow is an isomorphism, where $\rho^{+}=\left.\rho\right|_{\left(T_{N}^{*} X\right)}$ and $\mathcal{N}^{+}$ is the $\mathcal{E}_{Y}$-module defined in Lemma 2.3 .

Proof. This follows from the definition of $\mathcal{N}^{+}$and Theorem 3.5.6 of [SKK, Ch. II].

Q.E.D.

Since $\mathcal{N}^{+}$is coherent over $\mathcal{E}_{Y}$ and $\rho^{+}$is finite on $\operatorname{Supp}(\widetilde{\mathcal{M}}) \cap\left(T_{N}^{*} X\right)^{+}$, by Lemma 2.4, we have

$$
\begin{aligned}
\operatorname{RHom}_{\mathcal{E}_{Y}}\left(\mathcal{N}^{+}, \mathcal{C}_{N}\right) & \cong \operatorname{RHom}_{\mathcal{E}_{Y}}\left(\mathcal{N}^{+}, \mathcal{E}_{Y}\right) \otimes_{\mathcal{E}_{Y}}^{\mathbb{L}} \mathcal{C}_{N} \\
& \cong \rho_{*}^{+}\left[\left.\operatorname{RHom}_{\mathcal{E}_{X}}\left(\widetilde{\mathcal{M}}, \mathcal{E}_{X \leftarrow Y}\right)\right|_{\left(T_{N}^{*} X\right)+}\right] \otimes_{\mathcal{E}_{Y}}^{\mathbb{L}} \mathcal{C}_{N}[1] \\
& \cong \rho_{*}^{+}\left[\left.\operatorname{RHom} \mathcal{E}_{X}\left(\widetilde{\mathcal{M}}, \mathcal{E}_{X \leftarrow Y}\right)\right|_{\left(T_{N}^{*} X\right)^{+}} \otimes_{\rho^{-1} \mathcal{E}_{Y}}^{\mathbb{L}} \rho^{-1} \mathcal{C}_{N}\right][1]
\end{aligned}
$$


Using the $\mathcal{E}_{X^{-}}$-linear map $\mathcal{E}_{X \leftarrow Y} \otimes_{\rho^{-1} \mathcal{E}_{Y}} \rho^{-1} \mathcal{C}_{N} \rightarrow \mathcal{C}_{N \mid X}[\mathrm{KK}, \mathrm{II}]$, we have

$$
\begin{aligned}
\operatorname{RHom}_{\mathcal{E}_{Y}}\left(\mathcal{N}^{+}, \mathcal{C}_{N}\right) & \\
& \cong \rho_{*}^{+}\left[\left.\operatorname{RHom}_{\mathcal{E}_{X}}\left(\widetilde{\mathcal{M}}, \mathcal{E}_{X \leftarrow Y} \otimes_{\rho^{-1}}^{\mathbb{L}} \mathcal{E}_{Y} \rho^{-1} \mathcal{C}_{N}\right)\right|_{\left(T_{N}^{*} X\right)^{+}}\right][1] \\
& \rightarrow \rho_{*}^{+}\left[\left.\operatorname{RHom}_{\mathcal{E}_{X}}\left(\widetilde{\mathcal{M}}, \mathcal{C}_{N \mid X}\right)\right|_{\left(T_{N}^{*} X\right)^{+}}\right][1] .
\end{aligned}
$$

Let $q \in T_{N}^{*} Y \backslash N$. For $k \in \mathbf{Z}$, looking at the stalk at $q$, we have from (2.4)

$$
\operatorname{Ext}_{\mathcal{E}_{Y}}^{k}\left(\mathcal{N}_{q}^{+}, \mathcal{C}_{N q}\right) \rightarrow \bigoplus_{p \in\left(T_{N}^{*} X\right)^{+} \cap \operatorname{Supp}(\mathcal{M}) \cap \rho^{-1}(q)} \operatorname{Ext}_{\mathcal{E}_{X}}^{k+1}\left(\widetilde{\mathcal{M}}_{p},\left(\mathcal{C}_{N \mid X}\right)_{p}\right) .
$$

It follows from the division theorem for the $\mathcal{E}_{X^{-}}$-module $\mathcal{C}_{N \mid X}[\mathrm{KK}, \mathrm{II}$, Proposition $3 ; \mathrm{KS} 2,6.3 .1]$ and the definition of $\mathcal{N}^{+}$that this is an isomorphism for any $k \in \mathbf{Z}$; therefore (2.4) is an isomorphism in $D^{b}\left(\dot{T}_{N}^{*} Y\right)$. This completes the proof of Theorem 1.2.

Proof of Theorem 1.4. Let $p \in H$. If $d f$ is micro-hyperbolic for $\mathcal{M}$ at $p$, we have

$$
\mathrm{R} \Gamma_{\pi^{-1} Z_{+}} \mathrm{RHom}_{\mathcal{D}_{X}}\left(\mathcal{M}, \mathcal{C}_{M}\right)_{p}=0
$$

([KS2, Theorem 2.2.1]). Since this holds at all $p \in H$ by hypothesis, we have an isomorphism

$$
\left.\left.\mathrm{R} \Gamma_{Z_{+}} \mathrm{RHom}_{\mathcal{D}_{X}}\left(\mathcal{M}, \mathcal{A}_{M}\right)\right|_{N} \stackrel{\sim}{\longrightarrow} \Gamma_{Z_{+}} \mathrm{RHom}_{\mathcal{D}_{X}}\left(\mathcal{M}, \mathcal{B}_{M}\right)\right|_{N} .
$$

Combining this and (1.4), we get (1.5).

Q.E.D.

\section{§3. Microlocal Boundary Value Problem}

We follow the notation of Section 1. By the proof given in Section 2, we have the following microlocal formula.

Theorem 3.1. Let $\Omega$ be an open conic subset of $T_{N}^{*} Y \backslash N$. Let $\rho$ : $T_{N}^{*} X \rightarrow T_{N}^{*} Y$, and let $\mathcal{M}$ be a coherent $\mathcal{E}_{X}$-module defined on $\rho^{-1}(\Omega)$. We assume

(1) $\varphi: Y \rightarrow X$ is non characteristic for $\mathcal{M}$ on a neighborhood of $\Omega$ in the sense of [SKK, Ch. II, Def. 3.5.4]

(2) $d f(p) \notin C\left(\operatorname{Supp}(\mathcal{M}), Z_{+} \times_{M} T_{M}^{*} X\right)$, at any point $p$ of $\rho^{-1}(\Omega) \cap T_{M}^{*} X$.

We then have the isomorphism

$$
\mathrm{R} \rho_{*} \mathrm{RHom} \mathcal{E}_{X}\left(\mathcal{M}, \mathcal{C}_{Z_{+} \mid X}\right) \otimes \operatorname{or}_{N \mid M}[1] \cong \mathrm{RHom}_{\mathcal{E}_{Y}}\left(\mathcal{N}^{+}, \mathcal{C}_{N}\right)
$$

on $\Omega$, where $\mathcal{N}^{+}$is the coherent $\mathcal{E}_{Y}$-module given by Lemma 2.3. 
To obtain the formula above by the proof of Section 2, we have to prove Lemma 2.2 for $\mathcal{E}_{X}$-modules, and we need to use the micro-support estimate

$$
\operatorname{SS}\left(\operatorname{RHom} \mathcal{E}_{X}\left(\mathcal{M}, \mu \operatorname{hom}\left(\mathbf{C}_{Z_{+}}, \mathcal{O}_{X}\right)\right) \subset C\left(\operatorname{Supp}(\mathcal{M}), \operatorname{SS}\left(Z_{+}\right)\right)\right.
$$

for coherent $\mathcal{E}_{X^{-}}$modules. (Note that $\mu$ hom $\left(\mathbf{C}_{Z_{+}}, \mathcal{O}_{X}\right)$ is an object of $D^{b}\left(\mathcal{E}_{X}\right)$.) This estimate can be obtained from the argument of 10.5.1 of [KS3] and Theorem 10.4.2. (We sketch the proof below.) If once we get the micro-support estimate above, we are able to prove Theorem 3.1 in the same way as in Section 2.

Sketch of Proof of (3.2). (See Sections 10.4 and 10.5 of [KS3] for the details.) We may assume that $\mathcal{M}$ is quasi-isomorphic to $\mathcal{E}_{X} \otimes_{\mathcal{E}(G ; D)} M$ for a bounded complex $M$ of free $\mathcal{E}(G ; D)$ modules of finite rank. For the ring $\mathcal{E}(G ; D)$, see Section 10.4.1 of [KS3] and [KS2]. Let $x \in D$, and $U$ a sufficiently small, $G$-round neighborhood of $x$. Let $\Omega_{0}$ and $\Omega_{1}$ be $G$-open sets in $X$ with $\Omega_{0} \subset \Omega_{1}$ and $\Omega_{1} \backslash \Omega_{0} \subset U$. We set

$$
\mathcal{F}=\left.\phi_{G}^{-1} \mathrm{R} \Gamma_{\Omega_{1} \backslash \Omega_{0}} \mathrm{R} \phi_{G *} \mathcal{O}_{X}\right|_{\Omega_{1}},
$$

with $\phi_{G}: X \rightarrow X_{G}$ the $G$-topology map. Then $\mathcal{F} \in D^{b}(\mathcal{E}(G ; D))$, and we have a canonical morphism $\mathcal{F} \rightarrow \mathcal{O}_{X}$ such that $\mathcal{F} \cong \mathcal{O}_{X}$ in $D^{b}\left(\Omega_{1} ; V\right)$, where

$$
V=\operatorname{Int}\left(\Omega_{1} \backslash \Omega_{0}\right) \times \operatorname{Int}\left(G^{\circ a}\right) .
$$

We then have

$$
\begin{aligned}
\left.\operatorname{RHom}_{\mathcal{E}_{X}}\left(\mathcal{M}, \mu \operatorname{hom}\left(\mathbf{C}_{Z_{+}}, \mathcal{O}_{X}\right)\right)\right|_{V} & \cong \operatorname{RHom} \\
& \left.\cong \mu \operatorname{hom}\left(\mathbf{C}_{Z_{+}}, \operatorname{RHom}_{\mathcal{E}(G ; D)}(M, \mathcal{F})\right)\right|_{V} .
\end{aligned}
$$

By applying Theorem 10.4.2, we have

$$
\operatorname{SS}\left(\operatorname{RHom}_{\mathcal{E}(G ; D)}(M, \mathcal{F})\right) \subset \operatorname{Supp}(\mathcal{M}) .
$$

Use the micro-support estimate for $\mu$ hom, and we get (3.2).

Q.E.D.

We say that $\mathcal{M}$ is locally semi-hyperbolic on $\Omega$ if $\mathcal{M}$ satisfies conditions (1) and (2) in Theorem 3.1. This can be described (in the case of $\mathcal{M}=\mathcal{E}_{X} / \mathcal{E}_{X} P$ ) in local coordinates as follows. Let $\left(x_{1}, \ldots, x_{n}\right)$ be a system of local coordinates of $M, Z_{+}=\left\{x_{1} \geq 0\right\}$. Let

$$
P=D_{1}^{m}+A_{1}\left(x, D^{\prime}\right) D_{1}^{m-1}+\cdots+A_{m}\left(x, D^{\prime}\right)
$$


be a micro-differential operator of order $m$, where $D^{\prime}=\left(D_{2}, \ldots, D_{n}\right)$, and let $\mathcal{M}=\mathcal{E}_{X} / \mathcal{E}_{X} P$. Let $(z, \zeta)$ denote the coordinates of $T^{*} X, z=x+i y$, and $\zeta=$ $\xi+i \eta$. We denote by $\tau=\tau_{1}\left(z, \zeta^{\prime}\right), \ldots, \tau_{m}\left(z, \zeta^{\prime}\right)$ the roots of $\sigma_{m}(P)\left(z ; \tau, \zeta^{\prime}\right)=0$. Then $\mathcal{M}$ is locally semi-hyperbolic on $\Omega$ if and only if, for every compact subset $K$ of $\Omega$, there are positive numbers $\delta$ and $C$ (depending on $K$ ) such that

$$
\operatorname{Re} \tau_{j}\left(z, \zeta^{\prime}\right) \leq C\left\{\left|\xi^{\prime}\right|+\left(|y|+\left(-x_{1}\right)_{+}\right)\left|\eta^{\prime}\right|\right\}
$$

holds for all $j=1, \ldots, m$ if $\left(x^{\prime}, i \eta^{\prime}\right) \in K,\left|x_{1}\right| \leq \delta,|y| \leq \delta,\left|\xi^{\prime}\right| \leq \delta\left|\eta^{\prime}\right|$, and $\left|\operatorname{Re} \tau_{j}\left(z, \zeta^{\prime}\right)\right| \leq \delta\left|\eta^{\prime}\right|$. Cf. [O, Section 3].

Now let $M_{+}=Z_{+} \backslash N$, and set

$$
\mathcal{C}_{M_{+} \mid X}=\mu \operatorname{hom}\left(\mathbf{C}_{M_{+}}, \mathcal{O}_{X}\right) \otimes \operatorname{or}_{M \mid X}[n]
$$

(cf. Schapira [S2]). This is an object of $D^{b}\left(\mathcal{E}_{X}\right)$.

If we identify or $_{N \mid M}$ with the constant sheaf $\mathbf{C}_{N}$ by the choice of an orientation, we have

Corollary 3.2. $\quad$ Assume (1) and (2) of Theorem 3.1. Let $M_{+}=Z_{+} \backslash N$. We have the isomorphism

$$
\mathrm{R} \rho_{*} \operatorname{RHom}_{\mathcal{E}_{X}}\left(\mathcal{M}, \mathcal{C}_{M_{+} \mid X}\right) \cong \operatorname{RHom} \mathcal{E}_{Y}\left(\mathcal{M}_{Y} / \mathcal{N}^{+}, \mathcal{C}_{N}\right)
$$

on $\Omega$, where $\mathcal{M}_{Y}$ is the induced $\mathcal{E}_{Y}$-module of $\mathcal{M}$, and $\mathcal{N}^{+}$the coherent $\mathcal{E}_{Y^{-}}$ submodule given by Lemma 2.3 .

Proof. This follows from (3.1) and Proposition 3 of [KK, II]. Q.E.D.

Remark. In getting (3.5), it is better to choose the isomorphism or ${ }_{N \mid M} \cong$ $\mathbf{C}_{N}$ so that

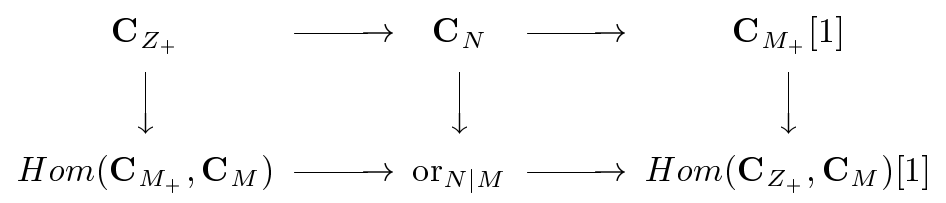

becomes commutative. (This corresponds to choosing a non-degenerate section $d f$ of $T_{N}^{*} M$ as positive orientation.) By this orientation, the isomorphism (3.5) becomes compatible with the boundary value morphism

$$
\mathrm{R} \rho_{*} \mathrm{RHom}_{\mathcal{E}_{X}}\left(\mathcal{M}, \mathcal{C}_{M_{+} \mid X}\right) \rightarrow \mathrm{RHom}_{\mathcal{E}_{Y}}\left(\mathcal{M}_{Y}, \mathcal{C}_{N}\right)
$$

constructed by Schapira [S2]. 
It will be useful in application to state Corollary 3.2 in the following form.

Corollary 3.3. $\quad$ Let $\Omega$ be as in Theorem 3.1. Let $\mathcal{M}$ be a coherent $\mathcal{E}_{X^{-}}$ module defined on $\rho^{-1}(\Omega)$ and satisfying condition $(1)$ in Theorem 3.1. Let $\mathcal{L}$ be a coherent quotient $\mathcal{E}_{X}$-module of $\mathcal{M}$. Assume that $\mathcal{L}$ satisfies condition (2) in Theorem 3.1. Let $Z$ be a closed conic subset of $\Omega$. We then have a commutative diagram

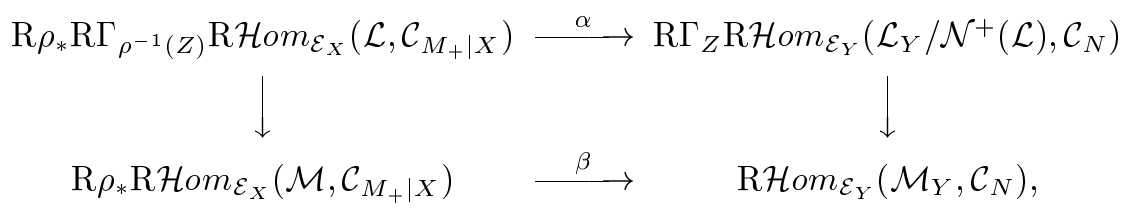

where $\mathcal{N}^{+}(\mathcal{L})$ denotes the coherent $\mathcal{E}_{Y}$-module induced from $\mathcal{L}$ by Lemma 2.3, and $\alpha$ is an isomorphism. ( $\beta$ is the microlocal boundary value morphism [S2].)

Let $\mathcal{M}, \mathcal{L}$ be as in Corollary 3.3. Assume also that there is a coherent $\mathcal{E}_{Y}$-module $\mathcal{N}$, and an $\mathcal{E}_{Y}$-linear map $\mathcal{N} \rightarrow \mathcal{M}_{Y}$ such that the composite $\mathcal{N} \rightarrow$ $\mathcal{M}_{Y} \rightarrow \mathcal{L}_{Y} / \mathcal{N}^{+}(\mathcal{L})$ is an isomorphism on $\Omega$. Then, by Corollary 3.3, there exists a morphism $\gamma$ such that

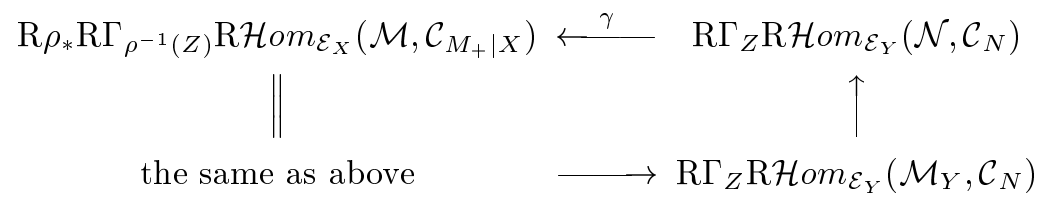

is commutative (if we start from $\mathrm{R} \Gamma_{Z} \mathrm{R} \mathcal{H} m_{\mathcal{E}_{Y}}\left(\mathcal{N}, \mathcal{C}_{N}\right)$ ). This recovers a result of Oaku [O, Theorem 3] in the case where $\mathcal{M}=\mathcal{E}_{X} / \mathcal{E}_{X} P$, with $P$ being a micro-differential operator of (3.3) (except that condition (3.4) is a little more restrictive than condition (C.1) of $[\mathrm{O}])$.

\section{References}

[KK] Kashiwara, M. and Kawai, T., On the boundary value problem for elliptic system of linear partial differential equations, I and II, Proc. Japan Acad., Ser. A, 48 (1972), 712-715. ibid., 49 (1973), 164-168.

[KS1] Kashiwara, M. and Schapira, P., Sheaves on Manifolds, Springer-Verlag, 1990.

[KS2] - Micro-hyperbolic systems, Acta Math., 142 (1979), 1-55.

[KS3] - Microlocal study of sheaves, Astérisque, 128 (1985), 1-235.

[Kt1] Kataoka, K., A microlocal approach to general boundary value problems, Publ. RIMS, Kyoto Univ., Suppl., 12 (1977), 147-153.

[Kt2] - Microlocal theory of boundary value problems, I and II, J. Fac. Sci. Univ. Tokyo, 27 (1980), 355-399. ibid., 28 (1981), 31-56. 
[Kt3] Kataoka, K., On the theory of Radon transformations of hyperfunctions, J. Fac. Sci. Univ. Tokyo, 28 (1981), 331-413.

[Kn] Kaneko, A., Singular spectrum of boundary values of solutions of partial differential equations with real analytic coefficients, Sci. Pap. Coll. Gen. Ed., Univ. Tokyo, 25 (1975), 59-68.

[O] Oaku, T., Microlocal Cauchy problems and local boundary value problems, Proc. Japan Acad., Ser. A, 55 (1979), 136-140.

[SKK] Sato, M., Kawai, T. and Kashiwara, M., Microfunctions and pseudo-differential equations, Lect. Notes Math., Springer, 287 (1973), 265-529.

[S1] Schapira, P., Propagation at the boundary and reflection of analytic singularities of solutions of linear partial differential equations, I, Publ. RIMS, Kyoto Univ., Suppl., 12 (1977), 441-453.

[S2] - Front d'onde analytique au bord, I, C. R. Acad. Sci., 302 (1986), 383-386.

[S3] - Microfunctions for boundary value problems, Algebraic Analysis, II (M. Kashiwara and T. Kawai, eds.), Academic Press, (1989), 809-819.

[SZ] Schapira, P. and Zampieri, G., Regularity at the boundary for systems of microdifferential operators, Hyperbolic Equations (F. Colombini and M. K. V. Murthy, eds.), Pitman Research Notes in Math., 158 (1987), 186-201.

[U] Uchida, M., RIMS Kōkyūroku, Kyoto Univ., 983 (1997), 156-166. 